\title{
Guía de Práctica Clínica: \\ Recomendaciones sobre el manejo de la diabetes
}

\author{
Recommendations for diabetes management
}

American Diabetes Association. Diabetes care, volume 33, supplement 1, january 2010.

Introducción

La Diabetes Mellitus (DM) es una enfermedad crónica cuya incidencia está en aumento, que requiere cuidados médicos continuos, así como educación profesional y del propio paciente para disminuir las complicaciones.

La Asociación Americana de Diabetes (ADA) ha publicado en el 2009 y actualizado en el 2010 la guía sobre rastreo, diagnós- tico y manejo de estos pacientes y de aquellos que pudieran desarrollarla.

\section{Clasificación y diagnóstico}

La guía encuadra a los pacientes en dos grupos: los diabéticos y los pacientes con riesgo aumentado de diabetes. Ver tabla 1

Tabla 1: clasificación de los pacientes de acuerdo a la guía de Asociación Norteamericana de Diabetes.

\begin{tabular}{|c|c|c|}
\hline Grupo & Criterios Diagnósticos (debe estar presente al menos uno de ellos) & Indicaciones de rastreo \\
\hline $\begin{array}{c}\text { Personas con diabetes mellitus } \\
1 \text { y } 2 \text { (DM1 y DM2) u otros tipos } \\
\text { (OTD) }\end{array}$ & $\begin{array}{l}\text { a) Glucemia en ayunas mayor a } 126 \mathrm{mg} / \mathrm{dL}^{\mathrm{a}} \text {. } \\
\text { b) Síntomas de hiperglucemia }{ }^{\mathrm{c}} \text { y glucemia casual mayor a } 200 \mathrm{mg} / \mathrm{dL}^{\mathrm{b}} \text {. } \\
\text { c) Glucemia mayor a } 200 \mathrm{mg} / \mathrm{dL}^{\mathrm{d}} \text { luego de dos horas de una carga de glucosa en el } \\
\text { contexto de una prueba de tolerancia oral la glucosa (PTOG). } \\
\text { d) Hemoglobina A1c (HA1c) mayor a } 6,5 \%{ }^{\mathrm{d}} \text {. }\end{array}$ & \multirow{2}{*}{$\begin{array}{l}\text { En adultos realizar glucemia en ayunas } \\
\text { ante sobrepeso (IMC } \geq 25 \text { ) ó al menos un } \\
\text { factor de FR cardiovascular y en mayores } \\
\text { de } 45 \text { sin FR. En niños realizar glucemia } \\
\text { en ayunas a partir de los diez años cada } \\
\text { tres años si hay sobrepeso (IMC > al } \\
\text { percentilo } 85 \text { para la edad y sexo ó } \\
\text { relación peso/talla mayor al P85) más } \\
\text { otros dos FR'. }\end{array}$} \\
\hline $\begin{array}{l}\text { Personas asintomáticas con } \\
\text { riesgo aumentado de diabetes }\end{array}$ & $\begin{array}{l}\text { a) Glucemia en ayunas alterada (GAA): glucemia entre } 100 \text { y } 125 \mathrm{mg} / \mathrm{dL} \text {. } \\
\text { b) Intolerancia a la glucosa (ITG): glucemia luego de dos horas de la PTOG entre } 140 \\
\text { y } 199 \mathrm{mg} / \mathrm{dL}^{\mathrm{d}} \text {. } \\
\text { c) Rango HA1c } 5,7 \text { a } 6,4 \% \text {. }\end{array}$ & \\
\hline
\end{tabular}

DM1: Diabetes tipo 1, DM2: Diabetes tipo 2, OTD: Otros tipos específicos de DM, DMG: Diabetes Gestacional. FR: factores de riesgo. IMC: índice de masa corporal. IMC Índice de masa corporal mayor o igual a $25 \mathrm{~kg} / \mathrm{m}^{2}$.

a Ayuno mínimo de ocho horas. ${ }^{\mathrm{b}}$ Cualquier momento del día sin importar el tiempo transcurrido desde la última comida. ${ }^{\circ}$ Poliuria, polidipsia y pérdida de peso inexplicada. ${ }^{d}$ En personas asintomáticas, estos criterios deben confirmarse con la repetición del mismo test en un día diferente. ${ }^{e}$ Este diagnóstico representa un factor de riesgo para el desarrollo de DM y enfermedad cardiovascular (ECV). ${ }^{\dagger}$ Sedentarismo, familiares de primer grado con DM, afroamericanos-latinos-americano nativo-americano asiático, diabetes mellitus gestacional (DMG), recién nacido mayor a 4 kg, Hipertensión arterial (HTA), HDL menor a 35mg/dL, triglicéridos mayores a $250 \mathrm{mg} / \mathrm{dL}$, síndrome de ovario poliquístico, GAA o ITG previo, acantosis nigricans, historia de enfermedad cardiovascular. ${ }^{9}$ Historia familiar de DBT2 en familiar de primero o segundo grado, raza/etnia (afroamericana-latina-americano asiático-americano) signos de insulinoresistencia o condiciones asociadas (acantosis nigricans-HTA-dislipemia-Síndrome de ovario poliquístico (SOP), pequeño o grande para la edad gestacional).

\section{Detección y diagnóstico de diabetes gestacional}

Se define diabetes mellitus gestacional (DMG) a cualquier grado de intolerancia a la glucosa que aparece o se reconoce por primera vez durante el embarazo. Su riesgo se valora en la primera consulta prenatal. Se clasifica en: muy alto riesgo para DMG -obesidad severa, historia previa de DMG o parto de un recién nacido $(\mathrm{RN})$ grande para edad gestacional, glucosuria, síndrome ovario poliquístico o historia familiar de DM tipo 2-, bajo riesgo para DMG (menos de 25 años edad, peso normal antes del embarazo, etnia de baja prevalencia, ausencia de historia de DM en familiar de primer grado, ausencia de historia de GAA, bajo riesgo de complicaciones obstétricas). El resto de las pacientes son consideradas como de riesgo intermedio. Las mujeres con muy alto riesgo de DMG deberían ser estudiadas con los test estándar para DM tan pronto como se confirme el embarazo. Para las pacientes de riesgo intermedio, incluidas aquellas que realizaron el test en forma temprana, se recomienda realizar el test para DMG entre las 24 y las 28 semanas de edad gestacional (EG). No se recomienda rastrear a las mujeres con bajo riesgo. El diagnóstico de DMG puede realizarse en dos pasos o en uno sólo.

Para la prueba en dos pasos se debe realizar entre las 24 y las 28 semanas una glucemia, luego de una hora de una carga oral de $50 \mathrm{~g}$ de glucosa. Si dicha glucemia es mayor a $140 \mathrm{mg} / \mathrm{dL}$ hay que realizar al día siguiente y con ocho horas de ayuno una PTOG con $100 \mathrm{~g}$ de glucosa.

Para la prueba en un paso, hay que realizar una PTOG con $100 \mathrm{~g}$ de glucosa entre las 24 y las 28 semanas, con ocho horas de ayuno, considerándose diagnóstico de DMG, al menos dos de los criterios siguientes: 1) Glucemia en ayunas mayor o igual a $95 \mathrm{mg} / \mathrm{dL}$; 2) durante la primera hora mayor o igual a $180 \mathrm{mg} / \mathrm{dL}$; 3) a las dos horas, $155 \mathrm{mg} / \mathrm{dL}$; 4) a las tres horas $140 \mathrm{mg} / \mathrm{dL}$.

Vale destacar que en Argentina se utiliza la PTOG con $75 \mathrm{mg}$ de acuerdo a las recomendaciones de la Sociedad Argentina de Diabetes.

Prevención de la diabetes mellitus (cambios en el estilo de vida y uso de metformina)

Se recomienda que las personas con ITG (A) y/ó GAA (E) realicen cambios en el estilo de vida (CEV) consistentes en una pérdida de un 5 a $10 \%$ de su peso y actividad física moderada (p. ej. una caminata de 150 minutos semanales). Podría utilizarse Metformina en aquellos pacientes con muy alto riesgo para desarrollar DM 2 (ITG y GAA asociados a A1C > 6\%, HTA, bajo HDL, triglicéridos elevados, familiar de primer grado con $D M)$ y en obesos menores de 60 años (E); recomendándose un control anual de estos pacientes.

\section{Manejo de los pacientes con diabetes mellitus tipo 2}

Se recomienda realizar una historia clínica completa, con laboratorio apropiado y el seguimiento por un equipo interdisciplinario.

Automonitoreo glucémico (AMG)

Se recomienda que los pacientes que usan múltiples dosis de insulina ó bomba de insulina realicen tres o más controles dia- 
rios (A); mientras que los pacientes que usan menos dosis diarias de insulina, antidiabéticos orales ó CEV solamente, el AMG puede usarse como guía de control del tratamiento (E). El monitoreo glucémico continuo (MGC) junto con el régimen de insulinización intensiva puede ser usado como una herramienta para descender la hemoglobina $\mathrm{A} 1 \mathrm{C}$ en adultos (mayores de 25 años) con DM1 (A)

Dosaje de hemoglobina glicosilada (HA1C)

Realizar un dosaje de HA1C al menos dos veces por año, en pacientes que reúnen los objetivos de buen control (E) y determinarla en forma trimestral en quienes requieren cambios en el tratamiento y no reúnen los objetivos de control (E). Los objetivos terapéuticos del paciente adulto con DM se resumen en el cuadro 1.

Cuadro 1: objetivos terapéuticos en adultos con diabetes mellitus.

- Hemoglobina glicosilada (HA1C) menor a 7\% (A)

- Hemoglucotest pre-prandial entre 70 y $130 \mathrm{mg} / \mathrm{dL}$.

- Pico de glucemia post-prandial (hemoglucotest) menor a 180 $\mathrm{mg} / \mathrm{dL}$

- En Diabetes gestacional glucemia en ayunas menor a 95 $\mathrm{mg} / \mathrm{dL}$, asociada a glucemia menor a $140 \mathrm{mg} / \mathrm{dL}$ un hora postprandial ó $120 \mathrm{mg} / \mathrm{dL}$ a las dos horas.

\section{Lineamientos generales de tratamiento de la diabetes mellitus}

Para los pacientes con DM1 se recomienda el tratamiento nutricional (TN) y el tratamiento con tres a cuatro dosis de insulina (basal y con las comidas); mientras que para los pacientes con DM2 se recomienda el TN más cambios de estilo de vida asociado a metformina y/o otros hipoglucemiantes orales si no se consiguen los objetivos. Los cuadros 2,3 y 4 resumen las recomendaciones nutricionales, de ejercicio y de vacunación de esta población.

Cuadro 2: tratamiento nutricional de los pacientes con diabetes mellitus y prediabetes.

- Plan alimentario individualizado (B).

- Disminución de un 5 a un $10 \%$ del peso en quienes tienen sobrepeso u obesidad (A).

- Ingesta de carbohidratos controlada (A).

- Menos de un $7 \%$ del total de calorías en grasas saturadas (A).

- Edulcorantes aprobados por FDA: acesulfame, aspartamo, neotame, sacarina y sucralosa.

- Endulzantes reducidos en calorías y alcoholes azucarados: eritriol-isomalt-lactitol-manitol-sorbitol-xylitol.

- Cirugía Bariátrica: considerarla en adultos con IMC mayor a $35 \mathrm{~kg} / \mathrm{m}^{2}$ y DM tipo 2, especialmente si es dificultoso el control con CEV y terapia farmacológica (B).

Cuadro 3: grandes conceptos sobre la prescripción de actividad física en los pacientes con diabetes.

- Se recomiendan 150 minutos por semana de actividad física aeróbica moderada a intensa, entendida ésta como la que conduce a que el paciente llegue al $50-70 \%$ de su frecuencia cardiaca máxima de acuerdo a su edad $(\mathrm{A})$.

- Aquellos pacientes con DM y retinopatía proliferativa o retinopatía no proliferativa severa no deben realizar ejercicios vigorosos, por el riesgo de hemorragia vítrea o desprendimiento de retina.

- Se recomienda actividades donde no se cargue peso (como natación, bicicleta) en aquellos pacientes con neuropatía periférica severa.
Cuadro 4: inmunizaciones recomendadas.

- Vacunación anual contra la influenza en todo paciente con DM mayor de seis meses de edad (C).

- Vacunación contra el neumococo para todo paciente diabético mayor de dos años de edad, con refuerzo en mayores de 65 años con inmunización previa y/o en menores de 65 años con inmunización hace más de cinco años (C).

\section{Rastreo y manejo de los factores de riesgo cardiovascular}

Se recomienda la valoración anual del riesgo cardiovascular a diez años tomando como predictores la dislipemia, la HTA, el consumo de tabaco, el antecedente de enfermedad vascular en familiar a edad temprana, y la micro o macroalbuminurias.

En los fumadores debe recomendarse firmemente el abandono del tabaquismo.

Si se trata de un paciente de alto riesgo se recomienda iniciar tratamiento con estatinas, aspirina en dosis antiagregante, e inhibidores de la enzima convertidora de angiotensina (IECA) o antagonistas del receptor de angiotensina II (ARAII) si requiere algún tratamiento antihipertensivo (B).

Se recomienda la toma de la tensión arterial (TA) en cada consulta. Si supera los $130 / 80 \mathrm{mmHg}$ en dos consultas se recomienda iniciar CEV y de persistir, agregar tratamiento con IECA ó ARAll, siendo el objetivo terapéutico una TA sistólica menor a $130 \mathrm{mmHg}(\mathrm{C})$ y una diastólica menor a $80 \mathrm{mmHg}(\mathrm{B})$. $\mathrm{Se}$ recomienda realizar al menos un control anual de lípidos. Si el colesterol LDL es menor a $100 \mathrm{mg} / \mathrm{dL}$, el HDL es mayor a 50 $\mathrm{mg} / \mathrm{dL}$ y los triglicéridos menores a $150 \mathrm{mg} / \mathrm{dL}$, puede espaciarse el control a cada dos años, además de ponerse énfasis en los CEV, el control del peso y la práctica de ejercicio (A). Respecto de las metas de colesterol LDL, en pacientes con enfermedad cardiovascular (ECV) establecida es un objetivo terapéutico llegar a menos de $70 \mathrm{mg} / \mathrm{dL}$ (B) y si no la hay, a 100 $\mathrm{mg} / \mathrm{dL}(\mathrm{A})$. Por otro lado, se busca un HDL mayor a $50 \mathrm{mg} / \mathrm{dL}$ en mujeres y de 40 en hombres, y en ambos sexos una trigliceridemia menor a $150 \mathrm{mg} / \mathrm{dL}$.

Si los objetivos no se alcanzan con estatinas a dosis máxima o si éstas no son toleradas, puede combinarse otro hipolipemiante para intentar lograr el descenso de lípidos deseado. También son candidatos al tratamiento con estatinas -más allá de sus valores de lípidos- quienes tienen ECV documentada o los pacientes menores de 40 años con uno o más factores de riesgo CV (A). Se recomienda el tratamiento antiplaquetario como prevención primaria (aspirina en dosis de 75 a $162 \mathrm{mg} / \mathrm{dL}$ ) en individuos con diabetes y riesgo cardiovascular elevado. En este grupo están incluidos los varones mayores de 50 años y las mujeres mayores de 60 años, con al menos un factor de riesgo mayor adicional. Para la prevención secundaria se utilizan las mismas dosis, existiendo una segunda línea de tratamiento con clopidogrel (B). Los pacientes con diabetes y ECV ya establecida deben recibir IECA (C) aspirina $(A)$ y estatinas $(A)$. Si el paciente ha tenido un infarto agudo de miocardio debe agregarse un Beta bloqueante para reducir su mortalidad.

Respecto de la metformina, puede utilizarse en individuos con insuficiencia cardíaca congestiva (ICC) estable y en ausencia de daño renal. Estos pacientes no deben recibir tiazolidinadionas (C).

\section{Rastreo y manejo del paciente con nefropatía}

En todos los pacientes se recomienda optimizar el control glucémico y el de la presión arterial $(A)$. Por otro lado, se recomienda controlar anualmente la excreción de albumina en 
personas con diabetes tipo 1 de más de cinco años de evolución y desde el momento del diagnóstico en quienes padecen diabetes tipo 2 (E). Con una determinación anual de la creatinina sérica se puede estimar el filtrado glomerular (GFR) y clasificar el estadío de la insuficiencia renal, si estuviera presente (E). El método de preferencia es la medición del cociente albuminuria/creatininuria en orina "spot". Se debe repetir dos a tres veces para confirmar el diagnóstico, pudiendo utilizarse también un dosaje de albuminuria en orina de 24 horas. La creatinina sérica se utiliza para evaluar la tasa de filtración glomerular y el estadio de daño renal a través de la fórmula de Cockroft-Gault ${ }^{\star *}$. LA tabla 2 resume los valores de referencia de protei-nuria en la orina "spot".

Tabla 2: valores de referencia de la proteinuria por el método de orina spot

\begin{tabular}{c|c}
\hline Normal & Menos de $30 \mathrm{ug} / \mathrm{mg}$ creatinina \\
\hline Microalbuminuria & 30 a $299 \mathrm{ug} / \mathrm{mg}$ creatinina \\
\hline Macroalbuminuria & Más de $300 \mathrm{ug} / \mathrm{mg}$ creatinina \\
\hline
\end{tabular}

Si existe micro o macroalbuminuria se prescribirán IECA ó ARAll (A) con monitoreo de la creatinina y el potasio, así como dietas con 0,8 a $1 \mathrm{~g} / \mathrm{kg} /$ día de proteínas en etapas tempranos de enfermedad renal y $0,8 \mathrm{~g} / \mathrm{kg} / \mathrm{día}$ en etapas avanzadas (B); con seguimiento con albuminuria para valorar la respuesta terapéutica. Si no queda clara la causa de la insuficiencia renal o ante dificultades con el tratamiento o progresión del daño renal se recomienda realizar una interconsulta con un nefrólogo, considerando además la prescripción de la vacunación contra hepatitis B.

\section{Rastreo y manejo del paciente con retinopatía}

Indicado anualmente o con mayor frecuencia si hay daño (B) en los pacientes con diabetes tipo 1 con más de cinco años de diagnóstico y en pacientes con diabetes tipo 2 desde el diagnóstico (B) En las mujeres que buscan embarazarse debe realizarse un examen oftalmológico previo, advirtiéndoles que tienen mayor riesgo de desarrollar o de que progrese la retinopatía; recomendándose en las embarazadas un examen durante el primer trimestre con seguimiento hasta un año luego del parto (B). También existe acuerdo en que el rastreo debería efectuarse a través de una retinografía realizada por profesionales entrenados $(E)$. Como tratamiento debe optimizarse el control de la glucemia y la presión arterial $(\mathrm{A})$ derivando al paciente a un oftalmólogo con experiencia ante la aparición de retinopatía o edema de mácula $(A)$ debiendo ofrecerse la fotocoagulación con láser para disminuir la chance de pérdida de la visón en los pacientes con retinopatía proliferativa, edema de mácula o casos severos de retinopatía no proliferativa (A). No está contraindicada la administración de aspirina en dosis antiagregante $(\mathrm{A})$.

\section{Rastreo y manejo del paciente con neuropatía}

Se recomienda una evaluación clínica anual para descartar polineuropatía periférica $(B)$ no siendo usualmente necesarias las pruebas electro-fisiológicas (E); y una evaluación clínica de neuropatía autonómica cardivascular a los cinco años del diagnóstico de diabetes tipo $1 \mathrm{y}$ al inicio del diagnóstico en el paciente con diabetes tipo $2(\mathrm{E})$.

Para el rastreo de polineuropatía simétrica periférica puede utilizarse una evaluación anual con un monofilamento de $10 \mathrm{~g}$ y diapasón de $128 \mathrm{~Hz}$, que tienen buena predicción de la aparición de úlceras. Para el rastreo de neuropatía autonómica debe interrogarse sobre intolerancia al ejercicio, hipotensión ortostática, taquicardia, constipación, gastroparesia, disfunción eréctil, disfunción genitourinaria, disfunción sudomotora, alteración neurovascular e hipoglucemias asintomáticas.

Ante la aparición de alguna de ellas debe optimizarse el control metabólico, evitar fluctuaciones con glucemias extremas, y si la polineuropatía es dolorosa, prescribir tratamiento farmacológico para aliviar los síntomas y mejorar la calidad de vida (E). Ver tabla 3.

\section{Cuidado de los pies}

Se recomienda efectuar una evaluación anual de los pies que incluya la toma de los pulsos, la inspección y el test del monofila-

Tabla 3: opciones farmacológicas para el tratamiento de la neuropatía diabética.

\begin{tabular}{|c|c|c|}
\hline Droga & Ejemplo & Dosis \\
\hline Antidepresivos tricíclicos & $\begin{array}{l}\text { Amitriptilina } \\
\text { Nortriptilina } \\
\text { Imipramina }\end{array}$ & $\begin{array}{l}10 \text { a } 75 \text { mg/día } \\
25 \text { a } 75 \mathrm{mg} / \text { día } \\
25 \text { a } 75 \mathrm{mg} / \text { día }\end{array}$ \\
\hline Anticonvulsivantes & $\begin{array}{c}\text { Gabapentina } \\
\text { Carbamazepina } \\
\text { Pregabalina }\end{array}$ & $\begin{array}{c}300 \text { a } 1200 \mathrm{mg} / \text { día } \\
200 \text { a } 400 \mathrm{mg} / \text { día } \\
60 \text { a } 120 \mathrm{mg} / \mathrm{dí} a\end{array}$ \\
\hline $\begin{array}{l}\text { 5-hidroxitriptamina e inhibidores de la } \\
\text { recantación de norepinefrina }\end{array}$ & Duloxetina & 60 a $120 \mathrm{mg} / \mathrm{dí} a$ \\
\hline Inhibidores de sustancia $P$ & Capsaicina (crema) & 0,025 a 1,075\% por aplicación \\
\hline
\end{tabular}

mento (B) así como brindar educación para la prevención de las úlceras (B) y seguimiento multidisciplinario en caso de que el paciente desarrolle dicha complicación (B). Si el paciente es fumador o tiene alguna anormalidad en la evaluación inicial se recomienda interconsultarlo con algún profesional con experiencia en el tema (C). Se recomienda realizar una evaluación vascular -pulsos, interrogatorio de claudicación intermitente, eventual cálculo de índice tobillo/brazo (ITB)- sobre todo en pacientes mayores de 50 años o menores con factores de riesgo (tabaco, HTA, dislipemia, o más de diez años de diabetes). Son factores de riesgo para el desarrollo de úlcera asociada a diabetes: amputación o úlceras previas, neuropatía periférica, deformidades del pie, enfermedad vascular periférica, alteraciones visuales, nefropatía diabética, pobre control glucémico y tabaquismo.

\section{Situaciones especiales}

Niños y adolescentes con Diabetes tipo 1

Los niños con diabetes poseen diferente sensibilidad a la insulina que los adultos, variabilidad en los cambios metabólicos relacionados con el crecimiento, menor capacidad de auto cuidado y

** Clearence de Creatinina:= [(140 - Edad en años) x Peso en kg]/[Creatinina plasmática x 72] x Factor de corrección. El factor de corrección es 1 en el varón y 0,85 en la mujer. Los valores normales son 95 a 154 en el hombre y 70 a 115 en la mujer. 
Tabla 4: objetivos metabólicos en pacientes con diabetes tipo 1 de acuerdo a la etapa de la vida del paciente.

\begin{tabular}{c|c|c|c|c}
\multirow{2}{*}{ Edad } & \multicolumn{2}{|c|}{ Valor de glucemia (mg/dL) } & Hemoglobina A1c (\%) & \multirow{2}{*}{ Justificación } \\
\cline { 2 - 5 } & Pre-prandial & Post-prandial & & Mayor vulnerabilidad a la hipoglucemia. \\
\hline 6 a 6 años & 100 a 180 & 110 a 200 & Entre 7,5 y 8,5 & Pocas complicaciones antes de pubertad. \\
\hline 13 a 19 años & 90 a 180 & 100 a 180 & Menor a 7,5 & $\begin{array}{c}\text { Mayor riesgo de hipoglucemias, pudiendo desearse } \\
\text { una Hba1c menor a 7\% si no son frecuentes. }\end{array}$ \\
\hline
\end{tabular}

mayor vulnerabilidad a las hipoglucemias; por lo tanto deben establecerse diferentes objetivos metabólicos (que se resumen en la tabla 4) según las etapas de la vida y la madurez sexual. Sin embargo, debe evaluarse cada caso individualmente, debiendo aumentarse los objetivos de glucemia y HA1c si las hipoglucemias son frecuentes. Los controles postprandiales se solicitarán cuando hay discrepancia entre los controles pre prandiales y la HA1c, y para ayudar a establecer el régimen de insulina necesario.

En estas etapas de la vida se recomienda luego de los diez años el rastreo anual con el cociente microalbuminuria/creati-nina en orina spot en pacientes con más de cinco años de diabetes $(E)$ recomendándose iniciar tratamiento con IECA si hay microalbuminuria (E). Respecto de la tensión arterial se recomienda intervenir si la presión diastólica o sistólica supe-ran el percentilo 90 a 95, iniciando dieta hiposódica y actividad física y si fuera necesario, tratamiento farmacológico (E). Los IECA son el tratamiento inicial de elección $(E)$ y el objetivo te-rapéutico debe ser una TA menor a al percentilo 90 para la edad, el sexo y la talla (E). Se recomienda realizar determinaciones de lípidos en los mayores de dos años de edad con antecedentes familiares de dislipemia, un evento cardiovascular antes de los 55 años, en quienes no puedan registrarse los antecedentes. Si los antecedentes son negativos pueden comenzar a dosarse en la pubertad $(E)$. Se recomienda un seguimiento cada cinco años sin son normales y anualmente si son anormales. El tratamiento inicial es la optimización de los valores de glucemia y las medidas higiénico dietéticas (E). Luego de los diez años de edad se prescribirán estatinas si el colesterol LDL supera los $160 \mathrm{mg} / \mathrm{gL}$ ó los $130 \mathrm{mg} / \mathrm{dl}$ en pacientes con otros factores de riesgo CV (E). En estos últimos se perseguirá una meta de LDL menor de 100 $\mathrm{mg} / \mathrm{dL}(\mathrm{E})$. Se recomienda realizar un rastreo anual de retinopatía luego de los diez años de edad en pacientes con más de tres a cinco años de diabetes (E). Otras consideraciones: rastreo de enfermedad celíaca al momento del diágnostico de DM (E) repitiendo el test si es ne-gativo y existe sospecha clínica por detención del crecimiento, bajo peso, pérdida de peso o síntomas sugestivos de dicha condición $(\mathrm{E})$; rastreo de hipotiroidismo con dosaje de tirotropina (TSH) cada uno o dos años o ante sospecha clínica y ATPO y ATGu al diagnóstico (E).

Niños y adolescentes con diabetes tipo 2

Su incidencia está en aumento. Si bien el diagnóstico diferencial con diabetes tipo 1 no siempre es fácil, es importante para el abordaje terapéutico, siendo necesario el rastreo de las comorbilidades (lípidos, microalbuminuria, control oftalmológico) a partir del diagnóstico de DM.

\section{Cuidado pre-concepcional}

Se recomienda lograr una HA1c menor a $7 \%$ ya que disminuye el riesgo de malformaciones (B) y dar consejo preconcepcional desde la pubertad (B). Si se decide buscar un embarazo, se recomienda el tratamiento previo de la retinopatía, la nefropatía y la neuropatía $(E)$ recordando que las estatinas, los IECA, los ARA II y la mayoría de los hipoglucemiantes no están recomendados durante el embarazo (E).

Diabetes en ancianos

Si tienen una expectativa de vida buena y son física y mentalmente sanos, deberán mantener los mismos objetivos metabólicos que las personas de menor edad, pudiendo ser estos más laxos ante otras situaciones clínicas. Es importante tener en cuenta en los ancianos son más lábiles a la deshidratación y al coma hiperosmolar (E). Deben también tratarse los otros factores de riesgo CV (E) y los métodos de rastreo deben ser individualizados $(\mathrm{E})$.

\section{Diabetes durante la internación hospitalaria}

Para los pacientes críticos se debe tratar la hiperglucemia a partir de $180 \mathrm{mg} / \mathrm{dll}$, con el objetivo de lograr un rango entre 140 y $180 \mathrm{mg} / \mathrm{dL}$ (A). En los pacientes no críticos la evidencia es menos contundente en relación a los niveles deseados de glucemia. Se recomienda solicitar Hba1c durante la internación si el paciente no la tiene dosada previamente $(E)$ y la gestión de un seguimiento luego de la internación si no existía el diagnóstico previo de diabetes (E).

\section{Comentario}

Estas guías nos brindan pautas para el diagnóstico, el control y el tratamiento de una enfermedad compleja, de la cual surgen evidencias nuevas para su manejo a gran velocidad. Como ejemplo y comparando la versión 2010 con la previa, cabe mencionar sólo algunos cambios: inclusión de la HbA1c como parámetro diagnóstico, y cambios en los criterios de indicación de aspirina para la prevención primaria de la enfermedad cardiovascular y en los objetivos glucémicos para el paciente crítico.

Como regla general, la corrección de la hiperglucemia sigue siendo esencial ${ }^{12}$, aunque el tratamiento multidisciplinario resulta indispensable para controlar otros factores de riesgo cardiovasculares, y para disminuir así las complicaciones asociadas a la enfermedad ${ }^{3}$. Los esfuerzos deberían centrarse en incrementar el número de pacientes que logren los objetivos metabólicos globales y los cambios actitudinales hacia estilos de vida más saludables, sin olvidar la necesidad de individualizar los tratamientos valorando la edad, las co-morbilidades, la expectativa de vida y las preferencias de los pacientes.

Andrea Péchin [ Instituto Megan, Santa Rosa, La Pampa. andrea.pechin @ gmail.com ]

Soledad Lovazzano [ Servicio de Endocrinología del Hospital Italiano de Buenos Aires. soledad.lovazzano@hospitalitaliano.org.ar ]

Recibido el 02/12/09 y aceptado el 3/05/10.

Péchin A y Lovazzano S. Recomendaciones sobre el manejo de la Diabetes.Evid Act Pract Ambul. 13(2). 60-64. Abr-Jun. 2010. Comentado de: ADA.Standars of medical care in Diabetes 2009.Diabetes Care, volume 32, Supplement 1, January 2009 y ADA.Summary of Revisions for the 2010 Clinical Practice Recommendations. Diabetes care, volume 33, supplement 1, january 2010.

\section{Referencias}

1. Stolar M. Glycemic control and complications in type 2 diabetes mellitus. Am J Med. 2010 Mar;123(3 Suppl):S3-11.

2. Nordwall M. Good glycemic control remains crucial in prevention of late diabetic complications--the Linköping Diabetes Complications Study. Pediatr Diabetes. 2009 May;10(3):168-76. Epub 2008 Oct 22.

3. Brown A. Managing highly insulin-resistant diabetes mellitus: weight loss approaches and medical management. Postgrad Med.2010 Jan;122(1):163-71. 